\title{
The most recent concepts for the management of bacterial and fungal infections in ICU
}

\author{
Matteo Bassetti ${ }^{1,4^{*}}$ (D) Garyfallia Poulakou ${ }^{2}$ and Marin H. Kollef ${ }^{3}$
}

(C) 2018 Springer-Verlag GmbH Germany, part of Springer Nature and ESICM

The pattern of infections globally is changing as a result of myriad factors including global warming, increasing antimicrobial resistance, and population migration patterns. In Europe large numbers of refugees from the Middle East, Africa and elsewhere have introduced multidrug-resistant (MDR) tuberculosis and MDR Gramnegative bacteria including Acinetobacter baumannii and methicillin-resistant Staphylococcus aureus (MRSA) [1]. Escherichia coli has the highest resistance rates (up to $38.1 \%$ ) against broad-spectrum cephalosporins in Southern and Eastern Europe, while carbapenem-resistant Klebsiella pneumoniae is also increasingly common $(59.4 \%$ in Greece, $34.3 \%$ in Italy, $20.5 \%$ in Romania and less than $2 \%$ in other EU countries) [1]. Carbapenem resistance has also been reported in more than $50 \%$ of A. baumannii isolates in Portugal, Greece, Italy, Cyprus, Romania and Bulgaria. The clinical importance of increasing prevalence of MDR bacterial infections was highlighted by a systematic review assessing the impact of non-covering (inappropriate) empirical antibiotic treatment (IEAT) [2]. Multi-covariate analyses demonstrated that the prevalence of any MDR pathogen, Acinetobacter spp. specifically, and more recent study years were associated with IEAT. MDR rates were independently associated with mortality and the prevalence of MDRs was associated with IEAT [2].

The prevalence of infection in critically ill patients caused by MDR bacteria is rapidly evolving. Clinical studies aimed at improving our understanding of

*Correspondence: matteo.bassetti@asuiud.sanita.fvg.it

${ }^{4}$ Clinica Malattie Infettive, Azienda Sanitaria Universitaria Integrata di Udine, Presidio Ospedaliero Universitario Santa Maria della Misericordia, Piazzale Santa Maria della Misericordia 15, 33100 Udine, Italy

Full author information is available at the end of the article the changing patterns of these infections are urgently needed. A recent article summarizing the discussion of a round table meeting organized by the European Society of Intensive Care Medicine (ESICM) and European Society of Clinical Microbiology and Infectious Diseases (ESCMID) highlighted list priorities in the management of MDR pathogens, identified areas that urgently require more research and made recommendations that should be implemented today [3]. Ideal antibiotic utilization is an area of uncertainty requiring additional investigations aimed at better understanding dose optimization, duration of therapy, use of combination treatment, aerosolized antibiotics and the integration of rapid diagnostics as a guide for treatment [4]. Recent advancements in laboratory techniques allow for the rapid identification of the infecting pathogen and antibiotic susceptibility testing that may guide fine-tuning of empirically selected regimens, facilitate de-escalation of unnecessary antimicrobials and support infection control decisions [5]. To combat the issue of escalating antimicrobial resistance and IEAT, combination antimicrobial regimens have become increasing recommended for empiric and definitive therapy, especially for carbapenemase-producing K. pneumoniae [6]. There is also moderate to limited evidence in support of combination therapy for treating carbapenemase-producing $A$. baumannii infections, including pneumonia and bacteraemia [7]. One major issue in the spread of Gram-negative MDR bacteria is the overuse of carbapenems for empirical therapy that creates selective pressure for development of carbapenem resistance. Recently there has been a reappraisal of old compounds and a number of new drugs that will be important in the treatment of selected MDR pathogens and allow intensivists the possibility for "sparing" carbapenems. By definition, extended spectrum $\beta$-lactamases

\section{勿


(ESBLs) are inhibited by $\beta$-lactamase inhibitors (BLBLIs) such as clavulanate and tazobactam. Because of this characteristic, $\beta$-lactam $/ \beta$-lactamase inhibitors have been evaluated in several observational studies as carbapenem-sparing therapy for ESBL-producing bacterial infection. The MERINO trial was designed to help resolve the controversy of carbapenem vs piperacillin-tazobactam in the treatment of ESBL infections. The results make clear that piperacillin-tazobactam should no longer be considered an alternative to meropenem for empirical or definitive treatment of bloodstream infection due to ESBL infections [8].

Moreover, the presence of severe influenza continues to result in appreciable mortality especially among patients with compromised cardiopulmonary status and has been reported as a risk factor for invasive pulmonary aspergillosis and secondary bacterial infection with MDR pathogens even in non-immunocompromised patients [1].

Catheter and device-related infections represent a significant part of hospital-acquired infections, mostly due to the formation of biofilms. Hand hygiene and skin antisepsis for catheter insertion and handling remain the milestones of prevention of catheter colonisation [9]. Superiority of skin disinfection with chlorhexidine-alcohol was further potentiated with a new randomised controlled trial in 16 intensive care units, from Japan [10]. In the latter, both $0.5 \%$ and $1.0 \%$ alcohol chlorhexidine gluconate were superior to $10 \%$ aqueous povidone iodine for the prevention of intravascular catheter colonisation. In a high volume burn unit in the USA, a sustained reduction of central-line-associated bloodstream infection (CLABSI) rates from 15.5 per 1000 central-line days to zero over 3 years became feasible, with the implementation of a rigorous program translating research into practice including among others chlorhexidine bathing, chlorhexidine dressings and use of alcohol-impregnated caps [11].

Novel insights into biofilms will in the near future enable targeting of mature biofilms with short-course catheter locks for catheter salvage, with antibiotic adjuvants or enzymes (DispersinB, DNaseI) or cations chelators (EDTA), or molecules that directly target persister cells (sugars, ADEP4, antimicrobial peptides, etc.) [1]. As another glimpse into the future, phage therapy and passive immunization could improve our ability to treat biofilm-forming bacteria [5]. Direct molecular detection of pathogens in blood is anticipated as a breakthrough in microbiological diagnosis of severe infections. A multicentre, open-label, cluster-randomised crossover trial (EVAMICA) showed that the addition of direct molecular detection of pathogens in the blood with a commercial test to standard care improved microbiological diagnosis in patients with severe sepsis and shortened the time to start a species-specific antimicrobial therapy [12]. Finally, therapeutic drug monitoring (TDM) provides a shift to refined dosing strategies for antimicrobials, compared to standard dosing, which is warranted to enhance the probability of achieving adequate drug exposure and clinical success [13].

Invasive fungal infections (IFIs) are an increasingly frequent cause of severe infections with mortality that ranges between $30 \%$ and $40 \%$ in episodes occurring in critically ill patients [14]. Approximately $80 \%$ of IFIs are due to Candida species that remains the predominant pathogen in ICU. Over the past decades, incidence of invasive candidiasis (IC) has shown progressively increasing or stable rates in most regions [14, 15], probably as a result of the growing complexity of surgical procedures, the presence of patient populations at higher risk of infection and the changes in patient demographic characteristics [14]. While candidemia episodes are easier to recognize compared to deep-seated candidiasis, current recommendations mainly focus on the management of candidemia, while trials on deep forms such as intra-abdominal candidiasis (IAC) are lacking. Adequate antifungal treatment and source control, i.e. catheter removal during candidemia or abscess drainage in IAC, are independent predictors of reduced mortality [14, 16]. Mortality for IC should also be attributed to suboptimal diagnostic tools. Standard mycological conventional blood culture is a gold standard, but may fail to diagnose IC in up to $25-50 \%$, whereas time to positivity for yeast and species identification can take several days from the time the blood culture is drawn, depending on the implicated Candida species [16]. Unless antifungal therapy is promptly initiated by the confirmation of IC, effective antifungal therapy may be further delayed. In recent years, other non-culture-based tests for diagnosis and susceptibility have been advocated to offset the diagnostic challenges and improve patient management, but their availability is currently limited. BDG (beta-D-glucan) testing has shown high negative predictive value and should be useful to rule out IC; nevertheless, it is not specific for IC as it is considered a pan-fungal diagnostic method. Interestingly BDG showed an important value in differentiating IAC from colonisation in critically ill patients with gastrointestinal abdominal problems [17]. Over the last decade, early antifungal agents have been prescribed in adult patients admitted to the ICU for various purposes corresponding to prophylaxis or pre-emptive or empirical therapies. Various definitions have been proposed for pre-emptive therapy over the last 10 years. Consequently, the concept of pre-emptive strategy has been an area of confusion as treated patients have been described by some authors as having received empirical therapy while others refer to pre-emptive or presumptive 
Table 1 The 10 most remarkable rules for antifungal stewardship programmes in ICU

Restrict or avoid antifungal prophylaxis particularly with fluconazole
Differentiate infection from colonisation and do not treat colonisation
Use non-culture-based diagnostics for early detection of IC (BDG, CAGTA, PCR)
Limit the use of empirical therapy just based on risk factors
Promote early pre-emptive antifungal treatment based on risk factors, (heavy) colonisation and biomarkers
Get treatment right the first time with adequate drugs, mainly echinocandins
Have adequate source control within $48 \mathrm{~h}$ (catheter removal, appropriate drainage, surgical control)
Use an adequate dose: low dose is associated with resistance
De-escalate whenever possible (if possible, within 5 days) from echinocandins to azoles
Stop early useless therapy and check duration of therapy (no more than 7-10 days for pre-emptive)

CAGTA Candida albicans germ tube antibody, IC invasive candidiasis, BDG Beta-D-Glucan, PCR Polymerase Chain Reaction

therapy. This confusion has lead to overtreatment with the potential detrimental impact of fluconazole on Candida ecology in ICU and/or on Candida susceptibility to antifungal drugs [18]. The ESCMID guidelines no longer consider fluconazole to be the drug of choice for invasive candidiasis, and recommend the use of echinocandins as first-line empirical treatment [14]. The 2016 Infectious Diseases Society of America (IDSA) guidelines also prioritize echinocandins as first-line treatment prior to species identification and susceptibility testing. Favourable characteristics of echinocandins compared with fluconazole include fungicidal activity, broader spectrum of activity, excellent safety profile and few drug-drug interactions. However, despite growing evidence of the superiority of echinocandins and the emergence of resistance to fluconazole, especially among non-albicans strains of Candida, fluconazole is still widely used in clinical practice for the treatment of candidiasis. The optimal duration of intravenous therapy for IC has not been extensively studied. In a recent non-comparative trial, step-down to an oral azole was allowed after 5 days of intravenous treatment [19]. Early de-escalation, however, did not seem to impact survival. Although antimicrobial stewardship programmes are one of the highest priorities in healthcare systems, few refer to the implementation of antifungal stewardship and highlight specific points on which efforts should be focused. The most remarkable rules for developing antifungal stewardship programmes targeting antifungal treatment bundles are reported in Table 1.

\footnotetext{
Author details

${ }^{1}$ Department of Medicine, University of Udine and Azienda Sanitaria Universitaria Integrata, Udine, Italy. ${ }^{2}$ 3rd Department of Medicine, School of Medicine, Sotiria General Hospital of Athens, Athens National and Kapodistrian University, Athens, Greece. ${ }^{3}$ Division of Pulmonary and Critical Care Medicine, Washington University School of Medicine, St. Louis, MO, USA. ${ }^{4}$ Clinica Malattie Infettive, Azienda Sanitaria Universitaria Integrata di Udine, Presidio Ospedaliero Universitario Santa Maria della Misericordia, Piazzale Santa Maria della Misericordia 15, 33100 Udine, Italy.
}

Compliance with ethical standards

\section{Conflicts of interest}

Dr. Bassetti has participated in advisory boards and/or received speaker honoraria from Achaogen, Angelini, Astellas, Bayer, Basilea, Cidara, Gilead, Menarini, MSD, Paratek, Pfizer, The Medicine Company, Tetraphase and Vifor. The other authors declare no conflict of interest.

Received: 17 September 2018 Accepted: 29 September 2018 Published online: 6 October 2018

References

1. Dimopoulos G, Akova M (2017) An overview on severe infections in Europe. Intensive Care Med 43:686-689

2. Carrara E, Pfeffer I, Zusman O, Leibovici L, Paul M (2018) Determinants of inappropriate empirical antibiotic treatment: systematic review and meta-analysis. Int J Antimicrob Agents 51:548-553

3. De Waele JJ, Akova M, Antonelli M, Canton R, Carlet J, De Backer D, Dimopoulos G, Garnacho-Montero J, Kesecioglu J, Lipman J, Mer M, Paiva JA, Poljak M, Roberts JA, Rodriguez Bano J, Timsit JF, Zahar JR, Bassetti M (2018) Antimicrobial resistance and antibiotic stewardship programs in the ICU insistence and persistence in the fight against resistance. A position statement from ESICM/ESCMID/WAAAR round table on multi-drug resistance. Intensive Care Med 44(2):189-196

4. Kollef MH, Bassetti M, Francois B, Burnham J, Dimopoulos G, GarnachoMontero J, Lipman J, Luyt CE, Nicolau DP, Postma MJ, Torres A, Welte T, Wunderink RG (2017) The intensive care medicine research agenda on multidrug-resistant bacteria, antibiotics, and stewardship. Intensive Care Med 43:1187-1197

5. Bassetti M, Poulakou G, Ruppe E, Bouza E, Van Hal SJ, Brink A (2017) Antimicrobial resistance in the next 30 years, humankind, bugs and drugs: a visionary approach. Intensive Care Med 43:1464-1475

6. Bassetti M, Giacobbe DR, Giamarellou H, Viscoli C, Daikos GL, Dimopoulos G, De Rosa FG, Giamarellos-Bourboulis EJ, Rossolini GM, Righi E, Karaiskos I, Tumbarello M, Nicolau DP, Viale PL, Poulakou G, Critically III Patients Study Group of the European Society of Clinical Microbiology and Infectious Disease (ESCMID), Hellenic Society of Chemotherapy (HSC) and Società Italiana di Terapia Antinfettiva (SITA) (2018) Management of KPC-producing Klebsiella pneumoniae infections. Clin Microbiol Infect 24(2):133-144. https://doi.org/10.1016/j.cmi.2017.08.030

7. Garnacho-Montero J, Dimopoulos G, Poulakou G, Akova M, Cisneros JM, De Waele J, Petrosillo N, Seifert H, Timsit JF, Vila J, Zahar JR, Bassetti M, European Society of Intensive Care Medicine (2015) Task force on management and prevention of Acinetobacter baumannii infections in the ICU. Intensive Care Med 41(12):2057-2075. https://doi.org/10.1007/s0013 4-015-4079-4 
8. Harris PNA, Tambyah PA, Lye DC, Mo Y, Lee TH, Yilmaz M, Alenazi TH, Arabi Y, Falcone M, Bassetti M, Righi E, Rogers BA, Kanj S, Bhally H, Iredell J, Mendelson M, Boyles TH, Looke D, Miyakis S, Walls G, Al Khamis M, Zikri A, Crowe A, Ingram P, Daneman N, Griffin P, Athan E, Lorenc P, Baker P, Roberts L, Beatson SA, Peleg AY, Harris-Brown T, Paterson DL, MERINO Trial Investigators and the Australasian Society for Infectious Disease Clinical Research Network (ASID-CRN) (2018) Effect of piperacillin-tazobactam vs meropenem on 30-day mortality for patients with E coli or Klebsiella pneumoniae bloodstream infection and ceftriaxone resistance: a randomized clinical trial. JAMA 320(10):984-994. https://doi.org/10.1001/ jama.2018.12163

9. Beloin C, Fernández-Hidalgo N, Lebeaux D (2017) Understanding biofilm formation in intravascular device-related infections. Intensive Care Med 43(3):443-446. https://doi.org/10.1007/s00134-016-4480-7

10. Yasuda H, Sanui M, Abe T, Japanese Society of Education for Physicians and Trainees in Intensive Care (JSEPTIC) Clinical Trial Group et al (2017) Comparison of the efficacy of three topical antiseptic solutions for the prevention of catheter colonization: a multicenter randomized controlled study. Crit Care 21(1):320. https://doi.org/10.1186/s13054-017-1890-z

11. Sood G, Caffrey J, Krout K et al (2017) Use of implementation science for a sustained reduction of central-line-associated bloodstream infections in a high-volume, regional burn unit. Infect Control Hosp Epidemiol 38(11):1306-1311. https://doi.org/10.1017/ice.2017.191

12. Cambau E, Durand-Zaleski I, Bretagne S, Brun-Buisson C, Cordonnier C, Duval X, Herwegh S, Pottecher J, Courcol R, Bastuji-Garin S, EVAMICA study team (2017) Performance and economic evaluation of the molecular detection of pathogens for patients with severe infections: the EVAMICA open-label, cluster-randomised, interventional crossover trial. Intensive Care Med 43(11):1613-1625. https://doi.org/10.1007/s0013 4-017-4766-4

13. Tängdén T, Ramos Martín V et al (2017) The role of infection models and PK/PD modelling for optimising care of critically ill patients with severe infections. Intensive Care Med 7:1021-1032. https://doi.org/10.1007/ s00134-017-4780-6

14. Bassetti M, Garnacho-Montero J, Calandra T, Kullberg B, Dimopoulos G, Azoulay E, Chakrabarti A, Kett D, Leon C, Ostrosky-Zeichner L, Sanguinetti
M, Timsit JF, Richardson MD, Shorr A, Cornely OA (2017) Intensive care medicine research agenda on invasive fungal infection in critically ill patients. Intensive Care Med 43(9):1225-1238. https://doi.org/10.1007/ s00134-017-4731-2

15. Chakrabarti A, Sood P, Rudramurthy SM, Chen S, Kaur H, Capoor M, Chhina D, Rao R, Eshwara VK, Xess I, Kindo AJ, Umabala P, Savio J, Patel A, Ray U, Mohan S, lyer R, Chander J, Arora A, Sardana R, Roy I, Appalaraju B, Sharma A, Shetty A, Khanna N, Marak R, Biswas S, Das S, Harish BN, Joshi S, Mendiratta D (2015) Incidence, characteristics and outcome of ICUacquired candidemia in India. Intensive Care Med 41 (2):285-295

16. Bassetti M, Righi E, Ansaldi F, Merelli M, Scarparo C, Antonelli M, Garnacho-Montero J, Diaz-Martin A, Palacios-Garcia I, Luzzati R, Rosin C, Lagunes L, Rello J, Almirante B, Scotton PG, Baldin G, Dimopoulos G, Nucci M, Munoz P, Vena A, Bouza E, de Egea V, Colombo AL, Tascini C, Menichetti F, Tagliaferri E, Brugnaro P, Sanguinetti M, Mesini A, Sganga G, Viscoli C, Tumbarello M (2015) A multicenter multinational study of abdominal candidiasis: epidemiology, outcomes and predictors of mortality. Intensive Care Med 41(9):1601-1610. https://doi.org/10.1007/s0013 4-015-3866-2

17. León C, Ruiz-Santana S, Saavedra P, Castro C, Ubeda A, Loza A, MartínMazuelos E, Blanco A, Jerez V, Ballús J, Alvarez-Rocha L, Utande-Vázquez A, Fariñas O (2012) Value of $\beta$-D-glucan and Candida albicans germ tube antibody for discriminating between Candida colonization and invasive candidiasis in patients with severe abdominal conditions. Intensive Care Med 38(8):1315-1325. https://doi.org/10.1007/s00134-012-2616-y

18. Ferreira D, Grenouillet F, Blasco G, Samain E, Hénon T, Dussaucy A, Millon L, Mercier M, Pili-Floury S (2015) Outcomes associated with routine systemic antifungal therapy in critically ill patients with Candida colonization. Intensive Care Med 41(6):1077-1088. https://doi.org/10.1007/s0013 4-015-3791-4

19. Bailly S, Leroy O, Montravers P, Constantin JM, Dupont H, Guillemot D, Lortholary O, Mira JP, Perrigault PF, Gangneux JP, Azoulay E, Timsit JF (2015) Antifungal de-escalation was not associated with adverse outcome in critically ill patients treated for invasive candidiasis: post hoc analyses of the AmarCAND2 study data. Intensive Care Med 41(11):19311940. https://doi.org/10.1007/s00134-015-4053-1 